\title{
Resveratrol inhibits doxorubicin-induced cardiotoxicity via sirtuin 1 activation in H9c2 cardiomyocytes
}

\author{
MI-HUA LIU ${ }^{1 *}$, JIAN SHAN $^{2 *}$, JIAN LI $^{3^{*}}$, YUAN ZHANG $^{4}$ and XIAO-LONG LIN ${ }^{5}$ \\ ${ }^{1}$ Department of Clinical Laboratory, Affiliated Nanhua Hospital, University of South China, Hengyang, Hunan 421001; \\ ${ }^{2}$ Department of Pathology, Zhongshan Torch Development Zone Hospital, Zhongshan, Guangdong 528437; \\ ${ }^{3}$ Department of Ultrasonic Diagnosis, Bo'Ai Hospital of Zhongshan, Zhongshan, Guangdong 528403; \\ ${ }^{4}$ Department of Pathology, Mawangdui Hospital, Changsha, Hunan 410016; ${ }^{5}$ Department of Pathology, \\ The Third People's Hospital of Huizhou, Affiliated Huizhou Hospital of Guangzhou Medical University, \\ Huizhou, Guangdong 516002, P.R. China
}

Received February 27, 2015; Accepted April 25, 2016

DOI: $10.3892 /$ etm.2016.3437

\begin{abstract}
Doxorubicin (DOX) is an efficient drug used in cancer therapy; however, it can induce severe cytotoxicity, which limits its clinical application. In the present study, the effects of resveratrol (RES) on sirtuin 1 (SIRT1) activation in mediating DOX-induced cytotoxicity in $\mathrm{H} 9 \mathrm{c} 2$ cardiac cells was investigated. $\mathrm{H} 9 \mathrm{c} 2$ cells were exposed to $5 \mu \mathrm{M}$ DOX for $24 \mathrm{~h}$ to establish a model of DOX cardiotoxicity. Apoptosis of H9c2 cardiomyocytes was assessed using the MTT assay and Hoechst nuclear staining. The results demonstrated that pretreating H9c2 cells with RES prior to the exposure of DOX resulted in increased cell viability and a decreased quantity of apoptotic cells. Western blot analysis demonstrated that DOX decreased the expression level of SIRT1. These effects were significantly alleviated by co-treatment with RES. In addition, the results demonstrated that DOX administration amplified forkhead box O1 (FoxO1) and P53 expression levels in H9c2 cells. RES was also found to protect against DOX-induced increases of FoxO1 and P53 expression levels in H9c2 cells. Furthermore, the protective effects of RES were arrested by the SIRT1 inhibitor nicotinamide. In conclusion, the results demonstrated that RES protected $\mathrm{H} 9 \mathrm{c} 2$ cells against DOX-induced injuries via SIRT1 activation.
\end{abstract}

Correspondence to: Mr. Xiao-Long Lin, Department of Pathology, The Third People's Hospital of Huizhou, Affiliated Huizhou Hospital of Guangzhou Medical University, 1 Xuebei Street, Huizhou, Guangdong 516002, P.R. China

E-mail: 493814078@qq.com

*Contributed equally

Key words: resveratrol, doxorubicin, apoptosis, sirtuin 1, forkhead box protein $\mathrm{O} 1$

\section{Introduction}

Doxorubicin (DOX) remains one of the most widely used anticancer drugs, due to its potent therapeutic effects on various types of cancer (1). However, the clinical use of this valuable anticancer drug is limited by severe toxic side effects on the heart, which may result in heart failure (2). Numerous studies have implicated reactive oxygen species generation in the cardiotoxicity of DOX, which ultimately results in cardiomyocyte apoptosis $(3,4)$. A number of pharmacological interventions have been proposed as therapies to oppose DOX-induced cardiotoxicity, including resveratrol (RES) and oleanolic acid $(5,6)$.

RES is a polyphenol that is primarily found in red wine and possesses comprehensive physiological actions, including cardioprotective, anti-platelet and anti-inflammatory properties $(7,8)$. RES inhibits DOX-induced cardiotoxicity by reducing oxidative stress and decreasing the severity of cardiac dysfunction (9). In addition, the cardioprotective effect of RES is associated with the enhanced anti-cancer efficacy of DOX, as demonstrated in in vitro and in vivo studies $(10,11)$. Therefore, administering DOX in combination with RES may be a viable chemotherapeutic method to reduce DOX cardiotoxicity (12). However, the underlying mechanisms of the cardioprotective effect of RES in DOX-induced injuries are not fully elucidated.

Sirtuin 1 (SIRT1) is an aldehyde dehydrogenase-dependent class III histone deacetylase, which has been shown to extend the lifespan of model organisms and prevent apoptosis in mammalian cells (13). SIRT1 catalyzes the deacetylation of a number of proteins, generating nicotinamide (NAM) as a by-product that negatively regulates the activity of SIRT1 (14). Numerous studies have demonstrated that SIRT1 regulates cell defenses and survival in response to stress through a number of signaling pathways $(15,16)$. Luo et al $(17)$ demonstrated that, in response to oxidative stress and DNA damage, SIRT1 repressed p53-dependent apoptosis. In addition, Chen et al (18) indicated that RES inhibited hypoxia-induced apoptosis through the SIRT1-forkhead box O1 (FoxO1) signaling pathway in the H9c2 cardiac cell line. 
In the present study, $\mathrm{H} 9 \mathrm{c} 2$ cells were treated with $5 \mu \mathrm{M}$ DOX in order to establish a cardiotoxicity model induced by chemotherapy (19). The study aimed to investigate whether RES inhibits DOX-induced injuries through SIRT1 activation in $\mathrm{H} 9 \mathrm{c} 2$ cells.

\section{Materials and methods}

Reagents. MTT, Hoechst 33258, DOX, RES and NAM, a SIRT1 inhibitor, were purchased from Sigma-Aldrich (St. Louis, MO, USA). All cell culture medium components were purchased from Thermo Fisher Scientific, Inc. (Waltham, MA, USA), including Dulbecco's modified Eagle's medium (DMEM), fetal bovine serum (FBS), streptomycin and penicillin. The enhanced chemiluminescence (ECL) solution was purchased from Nanjing KeyGen Biotech Co., Ltd. (Nanjing, China).

Cell culture. H9c2 cardiac cells (Type Culture Collection of the Chinese Academy of Sciences, Shanghai, China) were cultured in DMEM supplemented with $10 \%$ FBS, $100 \mu \mathrm{g} / \mathrm{ml}$ streptomycin and $100 \mathrm{U} / \mathrm{ml}$ penicillin in a humidified atmosphere with $5 \% \mathrm{CO}_{2}$ at $37^{\circ} \mathrm{C}$. The $\mathrm{H} 9 \mathrm{c} 2$ cardiac myocytes were passaged every 2 days. Cells were seeded at a density of $2 \times 10^{6}$ cells/dish in $100 \mathrm{~mm}$ dishes with $10 \%$ FBS and incubated for $24 \mathrm{~h}$ at $37^{\circ} \mathrm{C}$. Next, the medium was changed to $0.5 \%$ FBS-supplemented DMEM for $24 \mathrm{~h}$ starvation. In order to select the appropriate concentration of RES for experiments, $\mathrm{H} 9 \mathrm{c} 2$ cells were pretreated with $5,10,25$ or $50 \mu \mathrm{M}$ RES for $24 \mathrm{~h}$. Furthermore, to select the appropriate RES incubation time, H9c2 cells were exposed to $25 \mu \mathrm{M}$ RES for $6,12,14$ or $48 \mathrm{~h}$. The different treatment groups of the H9c2 cardiac myocytes were as follows: Control cells, in which cells were treated with culture medium only; DOX-treated group, in which cells were treated with $5 \mu \mathrm{M}$ DOX for $24 \mathrm{~h}$; RES+DOX group, in which $\mathrm{H} 9 \mathrm{c} 2$ cardiac myocytes were pretreated with $25 \mu \mathrm{M}$ RES for $24 \mathrm{~h}$, followed by treatment with $5 \mu \mathrm{M}$ DOX for $24 \mathrm{~h}$; and the NAM+RES+DOX group, in which H9c2 cells were pretreated with $40 \mathrm{mM}$ NAM for $60 \mathrm{~min}$ before treatment with $25 \mu \mathrm{M}$ RES, followed by $5 \mu \mathrm{M}$ DOX.

MTT assay. The cell viability in the various treatment groups was assessed with an MTT assay. Prior to each experiment, H9c2 cardiac myocytes $(5,000$ cells/well) were seeded in 96-well microtiter plates. Following incubation with the SIRT1 inhibitor NAM $(40 \mathrm{mM})$ and/or RES $(25 \mu \mathrm{M})$ for $24 \mathrm{~h}$, the cells were treated with $5 \mu \mathrm{M}$ DOX for $24 \mathrm{~h}$. Subsequently, $10 \mu \mathrm{l}$ MTT solution was added to each well, and the plates were incubated for $4 \mathrm{~h}$ at $37^{\circ} \mathrm{C}$. The absorbance was measured at $470 \mathrm{~nm}$ using the SpectraMax 190 spectrophotometer (Molecular Devices LLC, Sunnyvale, CA, USA), and the optical density (OD) used to calculate the percentage of cell viability, as follows: Percentage of cell viability $(\%)=\left(\mathrm{OD}_{\text {treat }}\right.$ ment group $/ \mathrm{OD}$ control group $) \times 100$. Three independent experiments were performed under each experimental condition.

Hoechst 33258 nuclear staining for the assessment of apoptosis. Apoptosis was analyzed by fluorescence microscopy using the chromatin dye Hoechst 33258. Following the various treatments, the cells were fixed in ice-cold $4 \%$ paraformaldehyde dissolved in phosphate-buffered saline (PBS) at room temperature for $20 \mathrm{~min}$. Non-specific binding was blocked using 5\% normal goat serum (Gibco; Thermo Fisher Scientific, Inc.) in $0.01 \mathrm{M}$ PBS containing 0.3\% Triton X-100. Subsequently, the cells were washed twice with PBS and incubated with $10 \mu \mathrm{g} / \mathrm{ml}$ Hoechst 33258 for $15 \mathrm{~min}$ at room temperature in the dark. The cells were then visualized under a fluorescence microscope (BX50-FLA; Olympus Corporation, Tokyo, Japan). Apoptotic cells present condensed, fractured or distorted nuclei, whereas viable cells displayed normal nuclear size and uniform fluorescence. The percentage of apoptotic cells was calculated, as follows: Apoptotic cells $(\%)=$ number of apoptotic cells/(number of apoptotic cells + number of viable cells) x 100.

Western blot analysis. The cells were homogenized using cell lysis buffer (Cell Signaling Technology, Inc., Danvers, MA, USA), and the lysates were centrifuged at $12,000 \mathrm{x}$ for 10 min at $4^{\circ} \mathrm{C}$. Protein concentration was determined using a $\mathrm{BCA}$ protein assay kit according to the manufacturer's instructions. The extracted proteins were mixed with 5\% SDS-PAGE sample buffer, then boiled at $100^{\circ} \mathrm{C}$ for $7 \mathrm{~min}$ and separated by electrophoresis on a $10 \%$ SDS-polyacrylamide gel, in which glyceraldehyde 3-phosphate dehydrogenase (GAPDH) served as a loading control. Following electrophoresis, the proteins were transferred to polyvinylidene difluoride membranes. The membranes were blocked in Tris-buffered saline- $0.1 \%$ Tween 20 (TBS-T) containing 5\% non-fat dry milk for $2 \mathrm{~h}$ at room temperature with rotation. Subsequent to blocking, the membranes were incubated overnight at $4^{\circ} \mathrm{C}$ with the following primary antibodies: Rabbit anti-SIRT1 polyclonal antibody (Cell Signaling Technologies, Inc.; dilution, 1:2,000; cat. no. 9475), rabbit anti-FoxO1 monoclonal antibody (Cell Signaling Technologies, Inc.; dilution, 1:1,000; cat. no. 2880), rabbit anti-P53 monoclonal antibody (Abcam, Cambridge, UK; dilution, 1:2,000; cat. no. ab179477), rabbit anti-Bcl-2-like protein 11 (Bim) polyclonal antibody (Abcam; dilution, 1:200; cat. no. ab32158) and mouse anti-GAPDH monoclonal antibody (Beyotime Institute of Biotechnology, Haimen, China; dilution, 1:1,000; cat. no. AG019). Primary antibodies were removed by washing the membranes three times with TBST, and the membranes were then incubated for $2 \mathrm{~h}$ with horseradish peroxidase-conjugated goat anti-rabbit and goat anti-mouse $\mathrm{IgG}(\mathrm{H}+\mathrm{L})$ (Beyotime Institute of Biotechnology; dilution, 1:2,000; cat. nos. A0208 and A0216, respectively). The membranes were then washed three times in TBS-T, and the antigen-antibody bands were detected using ECL solution and visualized using X-ray film (Beyotime Institute of Biotechnology). Each experiment was repeated three times. For quantification, the films were scanned and analyzed using Image J version 1.47i software (National Institutes of Health, Bethesda, MA, USA).

Superoxide dismutase (SOD), malondialdehyde (MDA) and lactate dehydrogenase $(\mathrm{LDH})$ release assay. $\mathrm{H} 9 \mathrm{c} 2$ cardiomyocytes $\left(5 \times 10^{2}\right.$ cells/well) were seeded into 96-well microtiter plates. Following incubation with $40 \mathrm{mM}$ NAM and/or $25 \mu \mathrm{M}$ RES for $24 \mathrm{~h}$ at $37^{\circ} \mathrm{C}$, the cells were treated with $5 \mu \mathrm{M}$ DOX for $24 \mathrm{~h}$ at $37^{\circ} \mathrm{C}$. Subsequently, the supernatants were obtained by centrifugation at $12,00 \mathrm{x}$ g for $10 \mathrm{~min}$, after which kits were used to measure SOD, MDA and LDH activities (cat 


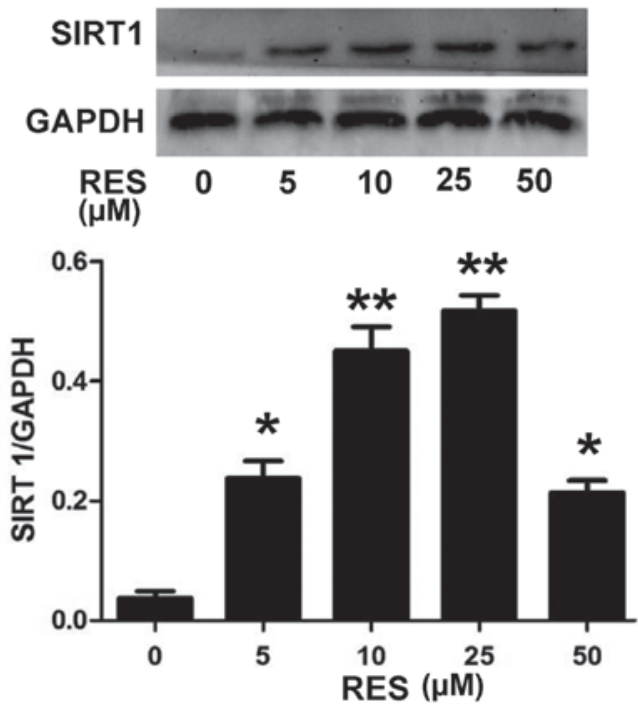

Figure 1. RES activates SIRT1 in H9c2 cells upon treatment with different concentrations. Data are shown as the mean \pm standard error $(n=3)$. ${ }^{*} \mathrm{P}<0.05$, ${ }^{* *} \mathrm{P}<0.01$ vs. the control group $(0 \mu \mathrm{M}$ RES $)$. SIRT1, sirtuin 1 ; RES, resveratrol.

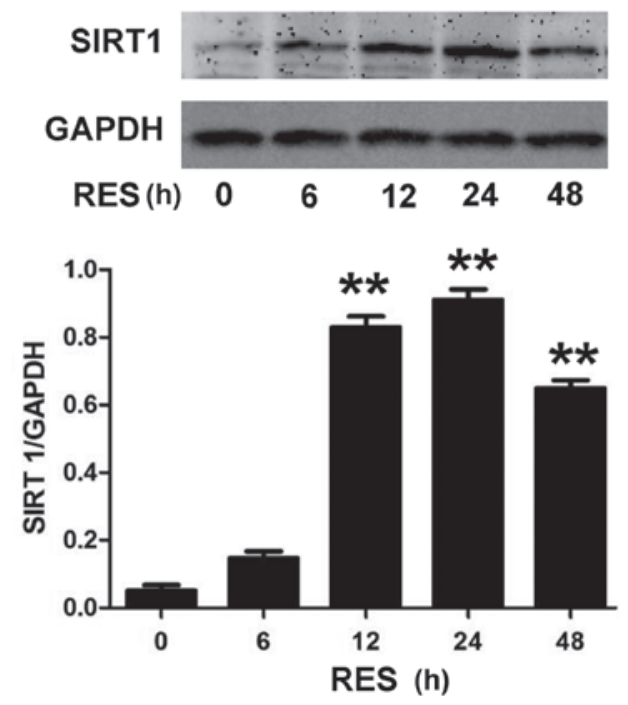

Figure 2. RES activates SIRT1 in H9c2 cells upon treatment with $25 \mu \mathrm{M}$ for different durations. Data are shown as the mean \pm standard error $(n=3)$. ${ }^{* * *} \mathrm{P}<0.01$ vs. the control group $(0 \mu \mathrm{M}$ RES $)$. SIRT1, sirtuin 1 ; RES, resveratrol.

nos. S0101, S0131 and C0016, respectively; Beyotime Institute of Biotechnology) according to the manufacturer's protocols.

Statistical analysis. The results are presented as the mean \pm standard error. Statistical analysis of the data was performed using Student's t-test or analysis of variance using SPSS version 13.0 (SPSS, Inc., Chicago, IL, USA). P $<0.05$ was considered to indicate a statistically significant difference.

\section{Results}

RES activates SIRT1 in H9c2 cells. In order to investigate the underlying mechanism of the anti-apoptotic effect of RES, the effect of RES on SIRT1 expression levels in H9c2 cells was investigated. As presented in Fig. 1, H9c2 cell pretreatment with RES at different concentrations $(5,10,25$ and $50 \mu \mathrm{M})$ for

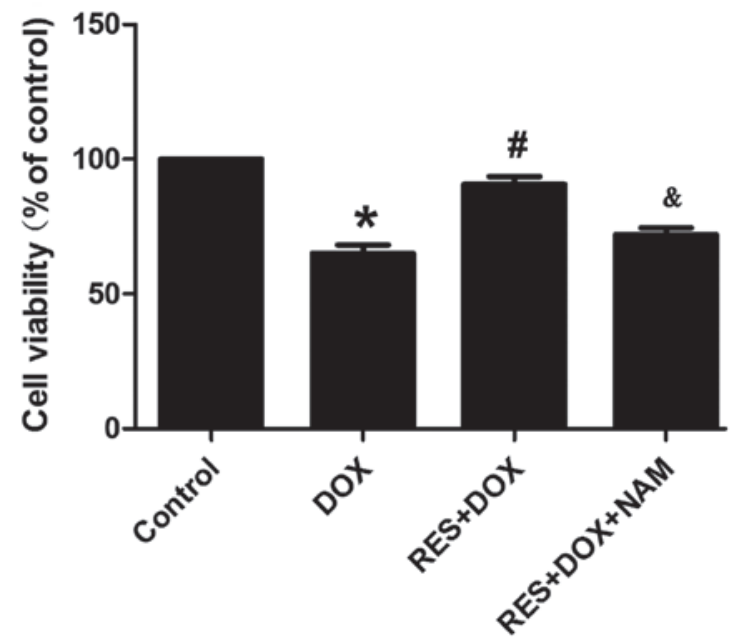

Figure 3. Resveratrol protects H9c2 cells against DOX-induced cytotoxicity. Cell viability was measured using an MTT assay. Data are shown as the mean \pm standard error $(n=3)$. ${ }^{~} \mathrm{P}<0.05$ vs. the control group; ${ }^{~} \mathrm{P}<0.05$ vs. the DOX-treated group; ${ }^{\&} \mathrm{P}<0.05$ vs. the RES+DOX group. DOX, doxorubicin; RES, resveratrol; NAM, nicotinamide.
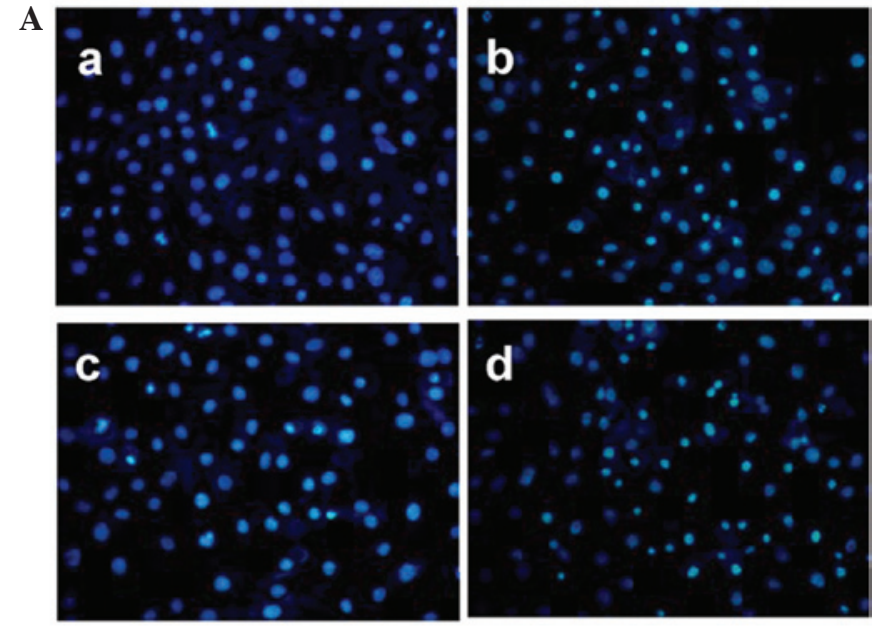

B

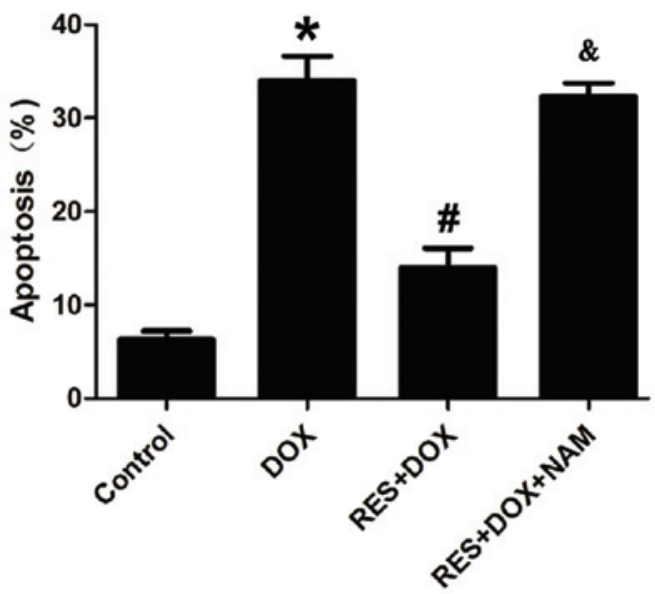

Figure 4. RES reduces DOX-induced apoptosis in H9c2 cells. (A) Hoechst 33258 nuclear staining followed by fluorescence imaging was used to investigate cell apoptosis in the various treatment groups: (Aa) Control group; (Ab) DOX group; (Ac) RES+DOX group; and (Ad) RES+DOX+NAM group. (B) The apoptotic rate was analyzed using a cell counter and Image $\mathbf{J}$ version 1.4 software. Data are shown as the mean \pm standard error $(n=5) .{ }^{~} P<0.05$ vs. the control group; ${ }^{~} \mathrm{P}<0.05$ vs. the DOX-treated group; ${ }^{\text {}} \mathrm{P}<0.05$ vs. the RES+DOX group. DOX, doxorubicin; RES, resveratrol; NAM, nicotinamide. 

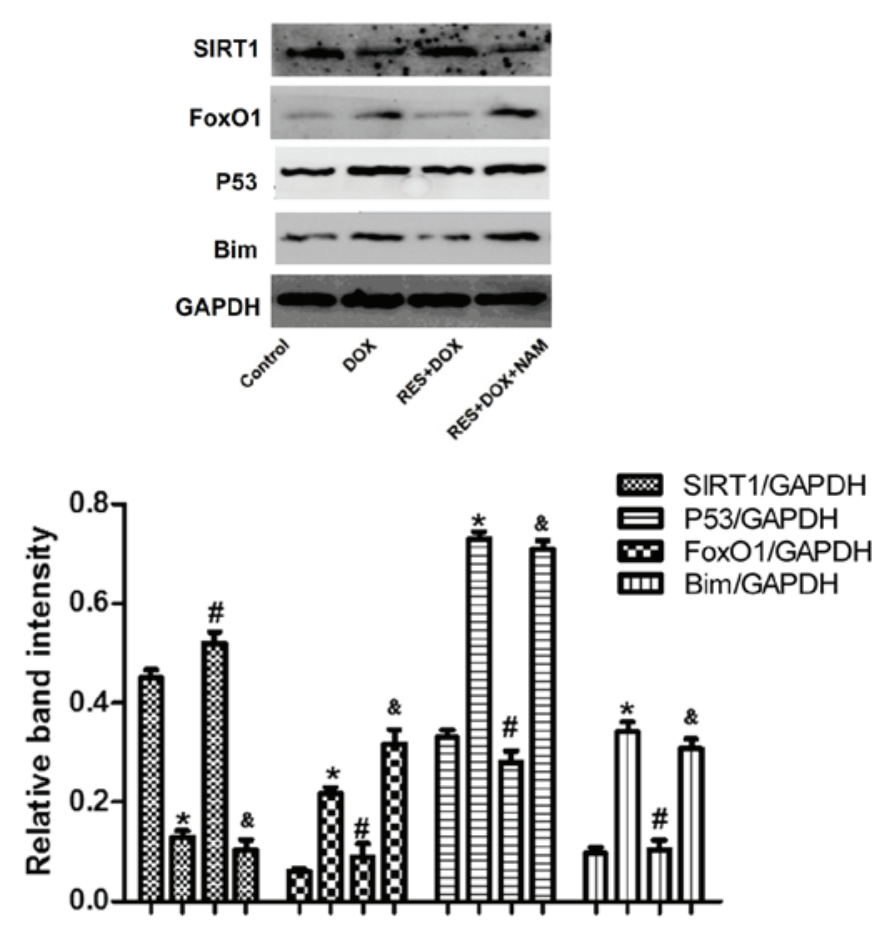

Figure 5. Protein expression levels of SIRT1, FoxO1, P53 and Bim in H9c2 cells treated with DOX, RES + DOX or NAM + RES + DOX, as analyzed by western blot analysis and quantified by densitometric analysis. Data are shown as the mean \pm standard error $(\mathrm{n}=3)$. ${ }^{*} \mathrm{P}<0.05$ vs. the control group; ${ }^{\#} \mathrm{P}<0.05$ vs. the DOX-treated group; ${ }^{\circledR} \mathrm{P}<0.05$ vs. the RES+DOX group. SIRT1, sirtuin 1; FoxO1, forkhead box protein O1; Bim, Bcl-2-like protein 11; DOX, doxorubicin; RES, resveratrol; NAM, nicotinamide.

$24 \mathrm{~h}$, prior to exposure to $5 \mu \mathrm{M}$ DOX for $24 \mathrm{~h}$, significantly increased the expression levels of SIRT1 $(\mathrm{P}<0.05$ or $\mathrm{P}<0.01)$. The expression levels of SIRT1 reached a peak following treatment with $25 \mu \mathrm{M}$ RES, and then declined when $50 \mu \mathrm{M}$ RES was used. In addition, as indicated in Fig. 2, exposure of H9c2 cells to $25 \mu \mathrm{M}$ RES for time periods ranging between 6 and $48 \mathrm{~h}$ resulted in the rapid activation of SIRT1. The SIRT1 expression was significantly higher at 12,24 and $48 \mathrm{~h}(\mathrm{P}<0.01)$ compared with the control ( $0 \mu \mathrm{M}$ RES), with the most notable effect at $24 \mathrm{~h}$. Therefore, the concentration of $25 \mu \mathrm{M}$ RES and incubation time of $24 \mathrm{~h}$ were selected for subsequent experiments.

RES inhibits DOX-induced cytotoxicity. Cell viability was investigated using MTT assay. As Fig. 3 demonstrates, the exposure of $\mathrm{H} 9 \mathrm{c} 2$ cells to DOX for $24 \mathrm{~h}$ induced significant cytotoxicity $(\mathrm{P}<0.05)$, resulting in a decrease in cell viability. However, cell pretreatment with $25 \mu \mathrm{M}$ RES for $24 \mathrm{~h}$ prior to exposure to DOX significantly ameliorated DOX-induced cytotoxicity $(\mathrm{P}<0.05)$, as evidenced by an increase in cell viability. Pretreatment of the H9c2 cells with $40 \mathrm{mM}$ NAM for 60 min prior to the exposure to RES plus DOX reversed the protective effect of RES, resulting in a significant decrease in cell viability $(\mathrm{P}<0.05)$. These results suggest that RES exerts a protective effect against DOX-induced cytotoxicity.

RES reduces DOX-induced apoptosis in $H 9 c 2$ cells. The effects of RES on DOX-induced apoptosis were also observed using Hoechst 33258 nuclear staining. Fig. 4 demonstrates
A

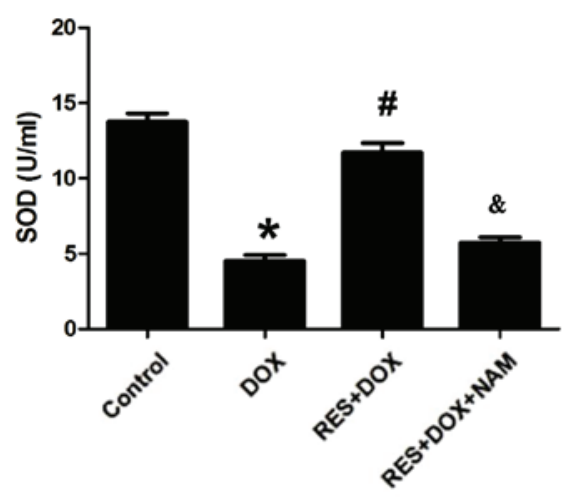

B

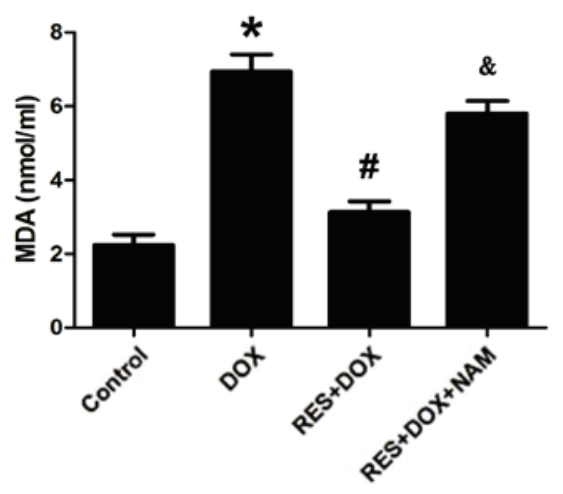

Figure 6. RES treatment prior to DOX restores SOD release and inhibits MDA production in $\mathrm{H} 9 \mathrm{c} 2$ cells, as shown by the (A) SOD activity and (B) MDA expression levels. Data are shown as the mean \pm standard error $(n=3)$. ${ }^{*} \mathrm{P}<0.05$ vs. the control group; ${ }^{\#} \mathrm{P}<0.05$ vs. the $\mathrm{DOX}$-treated group; ${ }^{\&} \mathrm{P}<0.05$ vs. the RES + DOX group. SOD, superoxide dismutase; MDA, malondialdehyde; DOX, doxorubicin; RES, resveratrol; NAM, nicotinamide.

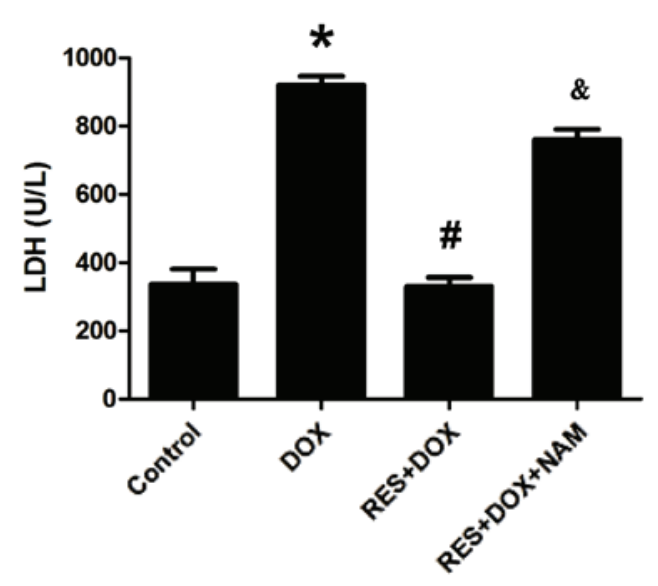

Figure 7. RES treatment prior to DOX inhibits LDH production in H9c2 cells, which is reversed upon pretreatment with NAM. Data are shown as the mean \pm standard error $(\mathrm{n}=3)$. ${ }^{*} \mathrm{P}<0.05$ vs. the control group; ${ }^{\#} \mathrm{P}<0.05$ vs. the DOX-treated group; ${ }^{\&} \mathrm{P}<0.05$ vs. the RES group. $\mathrm{LDH}$, lactate dehydrogenase; DOX, doxorubicin; RES, resveratrol; NAM, nicotinamide.

that $\mathrm{H} 9 \mathrm{c} 2$ cells treated with DOX for $24 \mathrm{~h}$ took up more Hoechst 33258 stain, as compared with the control group. However, cell pretreatment with RES for $24 \mathrm{~h}$ prior to DOX exposure significantly decreased the DOX-induced uptake of Hoechst $33258(\mathrm{P}<0.05)$. Pretreatment of the H9c2 cells with $40 \mathrm{mM}$ NAM for $60 \mathrm{~min}$ prior to the exposure of cells to RES plus DOX significantly reduced the protective effect of RES $(\mathrm{P}<0.05)$. 
SIRT1 inhibits DOX-induced apoptosis via the SIRTI signaling pathway. Studies have demonstrated that FoxO proteins induce the expression of cell death genes, such as Bim. In addition, FoxO proteins are primarily expressed in the cytoplasm, and are relocated to the nucleus when cells are subjected to various stresses; however, they can only interact with SIRT1 in the nucleus. Thus, SIRT1 can affect FoxO-induced transcription $(20,21)$. In the present study, pretreatment with $25 \mu \mathrm{M}$ RES for $24 \mathrm{~h}$ before administration of DOX was found to decrease the expression levels of FoxO1 and P53 (Fig. 5; P<0.05). However, this effect was reversed by the administration of $40 \mathrm{mM}$ NAM, prior to RES and DOX treatment. The expression of the FoxO target gene, Bim, was also investigated. It was observed that pretreatment of cells with RES inhibited the expression of Bim, and this effect was reversed by pretreatment with $40 \mathrm{mM}$ NAM, prior to RES and DOX.

RES restores SOD activity and decreases MDA expression levels in $\mathrm{H} 9 \mathrm{c} 2$ cells. In order to investigate the protective effects of RES, SOD activity and MDA expression levels in DOX-treated H9c2 cells were investigated as indicators of cell toxicity. As presented in Fig. 6, following treatment with DOX, the SOD activity was significantly decreased and the MDA expression level was significantly increased (both $\mathrm{P}<0.05$ ). However, pretreatment with RES significantly increased SOD activity and decreased the MDA expression (both $\mathrm{P}<0.05$ ). This protective effect of RES was reversed by the addition of 40 mM NAM $(\mathrm{P}<0.05)$.

RES decreases LDH levels in H9c2 cells. In order to investigate the protective effects of RES against DOX-induced cytotoxicity, the amount of LDH released into the culture medium was measured. As shown in Fig. 7, when cells were exposed to $\mathrm{DOX}, \mathrm{LDH}$ release was significantly increased $(\mathrm{P}<0.05)$; however, this was significantly reduced by pretreatment with RES $(\mathrm{P}<0.05)$. This protective effect of RES was significantly reversed following pretreatment with $40 \mathrm{mM}$ NAM $(\mathrm{P}<0.05$; Fig. 5).

\section{Discussion}

DOX is a widely used and successful anti-tumor drug; however, its clinical use is limited due to its severe cumulative dose-associated cardiotoxicity (2). Numerous studies have demonstrated that the primary molecular mechanism involved in DOX-induced cardiotoxicity is free radical-induced oxidative stress, and cardiomyocyte death by apoptosis and necrosis $(5,22)$. In accordance with previous studies $(23,24)$, in the present study it was observed that the exposure of H9c2 cells to DOX significantly induced cellular injuries, including decreases in cell viability and the expression of SIRT1, as well as increases in cell apoptosis and in the expression levels of FoxO1, P53 and Bim.

RES is a polyphenol that is primarily found in red wine, and occurs naturally in grapes, mulberries and peanuts. RES has numerous protective features; it can reduce the risk of cardiovascular disease and protect cardiomyocytes from apoptosis (25). It has also been demonstrated to protect cardiomyocytes against DOX-induced apoptosis (26). In addition,
RES has been shown to enhance the anti-cancer activity of DOX, and protect against DOX-induced cardiac toxicity in vitro and in mice (11). However, the mechanism underlying the effect of RES in protecting cardiomyocytes against apoptosis remains unclear.

It has been suggested that decreased SIRT1 expression is associated with DOX-induced myocardial apoptosis (27). As SIRT1 activity protects against increased oxidative stress and enhances cell survival, the SIRT1 signaling pathway is a plausible mechanism of action for the protective effect of RES against the effects of DOX. In the present study, the results demonstrated that SIRT1 expression was decreased following DOX treatment, and it was observed that RES preconditioning increased SIRT1 expression while significantly attenuating DOX-induced apoptosis. However, the effect of RES on SIRT1 expression levels was reduced in the presence of NAM.

Activated SIRT1 has been shown to protect the heart from oxidative stress by activating FoxO-dependent mechanisms (18). In addition, SIRT1 prevents p53-dependent apoptosis resistance to oxidative stress (28). Li et al (29) observed that RES protects cardiomyocytes from oxidative stress through the SIRT1 and mitochondrial biogenesis signaling pathways. Lou et al (30) reported that RES protects H9c2 cells against DOX-induced ER stress through ER stabilization, and significantly increased the activation of SIRT1, thereby leading to cardiac cell survival. To the best of our knowledge, the present study is the first to demonstrate that RES decreases the expression levels of FoxO1 and P53, and that this effect is largely reversed upon pretreatment with $40 \mathrm{mM}$ NAM. Furthermore, the effect of SIRT1 on a FoxO1-induced target gene expression was investigated in the current study. Consistently, RES reduced the expression of Bim and cell apoptosis. In addition, it was demonstrated that pretreatment of $\mathrm{H} 9 \mathrm{c} 2$ cells with $40 \mathrm{mM}$ NAM promoted the expression of Bim and H9c2 cell apoptosis.

In conclusion, the present study demonstrated that RES pretreatment is able to inhibit DOX-induced apoptosis in H9c2 cells and that its effects are mediated by SIRT1 activation. The current study investigated the mechanisms underlying the anti-apoptotic effect of RES in cardiomyocytes, and provided valuable evidence for identifying RES as a candidate for the treatment of cardiovascular diseases.

\section{Acknowledgements}

The present study was supported by the Graduate Student Research Innovation project of Hunan province (grant no. CX2013B397).

\section{References}

1. Menna P, Recalcati S, Cairo G and Minotti G: An introduction to the metabolic determinants of anthracycline cardiotoxicity. Cardiovasc Toxicol 7: 80-85, 2007.

2. Lipshultz SE, Karnik R, Sambatakos P, Franco VI, Ross SW and Miller TL: Anthracycline-related cardiotoxicity in childhood cancer survivors. Curr Opin Cardiol 29: 103-112, 2014.

3. Spallarossa P, Garibaldi S, Altieri P, Fabbi P, Manca V, Nasti S, Rossettin P, Ghigliotti G, Ballestrero A, Patrone F, et al: Carvedilol prevents doxorubicin-induced free radical release and apoptosis in cardiomyocytes in vitro. J Mol Cell Cardiol 37: 837-846, 2004. 
4. Lee DH, Kim S and Nam KS: Protective effects of deep sea water against doxorubicin-induced cardiotoxicity in H9c2 cardiac muscle cells. Int J Oncol 45: 2569-2575, 2014.

5. Al-Harthi SE, Alarabi OM, Ramadan WS, Alaama MN, Al-Kreathy HM, Damanhouri ZA, Khan LM and Osman AM: Amelioration of doxorubicin-induced cardiotoxicity by resveratrol. Mol Med Rep 10: 1455-1460, 2014.

6. Goyal SN, Mahajan UB, Chandrayan G, Kumawat VS, Kamble S, Patil P, Agrawal YO, Patil CR and Ojha S: Protective effect of oleanolic acid on oxidative injury and cellular abnormalities in doxorubicin induced cardiac toxicity in rats. Am J Transl Res 8: 60-69, 2016.

7. Renaud J, Bournival J, Zottig X and Martinoli MG: Resveratrol protects DAergic PC12 cells from high glucose-induced oxidative stress and apoptosis: Effect on p53 and GRP75 localization. Neurotox Res 25: 110-123, 2014.

8. Liu MH, Yuan C, He J, Tan TP, Wu SJ, Fu HY, Liu J, Yu S Chen YD, Le QF, et al: Resveratrol protects PC12 cells from high glucose-induced neurotoxicity via PI3K/Akt/FoxO3a pathway. Cell Mol Neurobiol 35: 513-522, 2014.

9. Tatlidede E, Sehirli O, Velioğlu-Oğünc A, Cetinel S, Yeğen BC, Yarat A, Süleymanoğlu S and Sener G: Resveratrol treatment protects against doxorubicin-induced cardiotoxicity by alleviating oxidative damage. Free Radic Res 43: 195-205, 2009.

10. Shankar S, Singh G and Srivastava RK: Chemoprevention by resveratrol: Molecular mechanisms and therapeutic potential. Front Biosci 12: 4839-4854, 2007.

11. Rezk YA, Balulad SS, Keller RS and Bennett JA: Use of resveratrol to improve the effectiveness of cisplatin and doxorubicin: Study in human gynecologic cancer cell lines and in rodent heart. Am J Obstet Gynecol 194: e23-e26, 2006.

12. Park DG: Antichemosensitizing effect of resveratrol in cotreatment with oxaliplatin in HCT116 colon cancer cell. Ann Surg Treat Res 86: 68-75, 2014.

13. Wang Y: Molecular links between caloric restriction and Sir2/SIRT1 Activation. Diabetes Metab J 38: 321-329, 2014.

14. Bitterman KJ, Anderson RM, Cohen HY, Latorre-Esteves M and Sinclair DA: Inhibition of silencing and accelerated aging by nicotinamide, a putative negative regulator of yeast sir 2 and human SIRT1. J Biol Chem 277: 45099-45107, 2002.

15. Alcendor RR, Gao S, Zhai P, Zablocki D, Holle E, Yu X, Tian B, Wagner T, Vatner SF and Sadoshima J: Sirtl regulates aging and resistance to oxidative stress in the heart. Circ Res 100 $1512-1521,2007$

16. Yamamoto T and Sadoshima J: Protection of the heart against ischemia/reperfusion by silent information regulator 1 . Trends Cardiovasc Med 21: 27-32, 2011.

17. Luo J, Nikolaev AY, Imai S, Chen D, Su F, Shiloh A, Guarente L and $\mathrm{Gu} \mathrm{W}$ : Negative control of p53 by Sir2alpha promotes cell survival under stress. Cell 107: 137-148, 2001.
18. Chen CJ, Yu W, Fu YC, Wang X, Li JL and Wang W: Resveratrol protects cardiomyocytes from hypoxia-induced apoptosis through the SIRT1-FoxO1 pathway. Biochem Biophys Res Commun 378: 389-393, 2009.

19. Guo R, Lin J, Xu W, Shen N, Mo L, Zhang C and Feng J: Hydrogen sulfide attenuates doxorubicin-induced cardiotoxicity by inhibition of the p38 MAPK pathway in $\mathrm{H} 9 \mathrm{c} 2$ cells. Int $\mathrm{J}$ Mol Med 31: 644-650, 2013.

20. Shukla S, Rizvi F, Raisuddin S and Kakkar P: FoxO proteins' nuclear retention and $\mathrm{BH} 3$-only protein Bim induction evoke mitochondrial dysfunction-mediated apoptosis in berberine-treated HepG2 cells. Free Radic Biol Med 76: 185-199, 2014.

21. Li F, Qu H, Cao HC, Li MH, Chen C, Chen XF, Yu B, Yu L, Zheng LM and Zhang W: Both FOXO3a and FOXO1 are involved in the HGF-protective pathway against apoptosis in endothelial cells. Cell Biol Int 39: 1131-1137, 2015.

22. Angsutararux P, Luanpitpong $S$ and Issaragrisil $S$ : Chemotherapy-Induced Cardiotoxicity: Overview of the Roles of Oxidative Stress. Oxid Med Cell Longev 2015: 795602, 2015.

23. Wang X, Wang XL, Chen HL, Wu D, Chen JX, Wang XX, Li RL, He JH, Mo L, Cen X, et al: Ghrelin inhibits doxorubicin cardiotoxicity by inhibiting excessive autophagy through AMPK and p38-MAPK. Biochem Pharmacol 88: 334-350, 2014.

24. Guo R, Wu K, Chen J, Mo L, Hua X, Zheng D, Chen P, Chen G, $\mathrm{Xu} \mathrm{W}$ and Feng J: Exogenous hydrogen sulfide protects against doxorubicin-induced inflammation and cytotoxicity by inhibiting p38MAPK/NFkB pathway in H9c2 cardiac cells. Cell Physiol Biochem 32: 1668-1680, 2013.

25. Das DK, Mukherjee S and Ray D: Erratum to: Resveratrol and red wine, healthy heart and longevity. Heart Fail Rev 16: 425-435, 2011

26. Oktem G, Uysal A, Oral O, Sezer ED, Olukman M, Erol A, Akgur SA and Bilir A: Resveratrol attenuates doxorubicin-induced cellular damage by modulating nitric oxide and apoptosis. Exp Toxicol Pathol 64: 471-479, 2012

27. Danz ED, Skramsted J, Henry N, Bennett JA and Keller RS: Resveratrol prevents doxorubicin cardiotoxicity through mitochondrial stabilization and the Sirtl pathway. Free Radic Bio Med 46: 1589-1597, 2009.

28. Shang L, Zhou H, Xia Y, Wang H, Gao G, Chen B, Liu Q, Shao C and Gong Y: Serum withdrawal up-regulates human SIRT1 gene expression in a p53-dependent manner. J Cell Mol Med 13: 4176-4184, 2009.

29. Li YG, Zhu W, Tao JP, Xin P, Liu MY, Li JB and Wei M: Resveratrol protects cardiomyocytes from oxidative stress through SIRT1 and mitochondrial biogenesis signaling pathways. Biochem Biophys Res Commun 438: 270-276, 2013.

30. Lou Y, Wang Z, Xu Y, Zhou P, Cao J, Li Y, Chen Y, Sun J and Fu L: Resveratrol prevents doxorubicin-induced cardiotoxicity in $\mathrm{H} 9 \mathrm{c} 2$ cells through the inhibition of endoplasmicreticulum stress and the activation of the Sirt1 pathway. Int J Mol Med 36: 873-880, 2015. 